\title{
A Google Trends Analysis of Facial Plastic Surgery Interest During the COVID-19 Pandemic
}

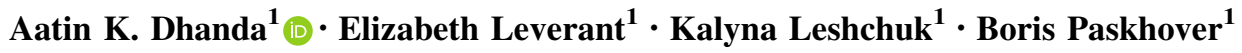

Received: 23 July 2020/ Accepted: 26 July 2020/Published online: 5 August 2020

(C) Springer Science+Business Media, LLC, part of Springer Nature and International Society of Aesthetic Plastic Surgery 2020

\begin{abstract}
The COVID-19 pandemic has had a multitude of effects on daily life. Aesthetic and cosmetic surgery practices have been significantly reduced in their working capacity or closed during this time. We used Google Trends to gauge the public's interest in facial plastic surgery during this pandemic, and how it has changed over the preceding months. As local shelter-in-place orders are being lifted, interest in facial plastic surgery is increasing even in the context of an ongoing national pandemic. Level of evidence $V$ Letter to the Editor.
\end{abstract}

Keywords COVID-19 - Google trends · Facial plastic surgery

The COVID-19 pandemic is ongoing in the USA and has had wide-ranging effects on daily life. Many nonessential or elective medical practices remain closed or are operating under reduced capacity. During times of duress, public interest in these elective procedures is understandably decreased. However, even though the pandemic is continuing and cases are still rising, interest in elective procedures as judged by search engine popularity has increased to pre-pandemic levels.

Google Trends is a tool provided by the search engine Google which analyzes the relative interest or popularity of a search term, on a scale from 0 to 100 with 100 being the highest popularity for a selected time range. The utility of

Aatin K. Dhanda

akd92@njms.rutgers.edu

1 Department of Otolaryngology Head and Neck Surgery, Rutgers - New Jersey Medical School, 90 Bergen Street, Suite 8100 , Newark, NJ 07103, USA
Google Trends has been shown before [1]. Using Google Trends, we analyzed 10 terms related to aesthetic plastic surgery or cosmetic medical procedures from January 1 2020 to July 102020 with a focus on facial plastic surgery.

As shown in Fig. 1, there was a noticeable decline in interest during March and April of 2020 relative to the first two months of the year. The average interest levels of all search terms for each month are shown in Fig. 2, demonstrating that overall interest dropped approximately 15 points from February to April. However, beginning in May, the relative interest began to rise, eventually exceeding levels during January and February. Some terms had large variances between months, such as "Botox" whose interest decreased by approximately 42 points from February to April (77.1-35.6). Other terms, like "Chin augmentation," saw only a 4-point decrease in the same time period. Of note, the increased interest in these terms has correlated well with increase in activity in our senior author's office, but this is purely commentary, and the experience of other facial plastic surgeons may be varied.

To provide some context to the timeline of this pandemic, the World Health Organization (WHO) declared COVID-19 as a pandemic on March 11 [2]. While restrictions vary widely across the country, New York state and specifically New York City are considered the epicenter of COVID in the USA. On March 20, New York State and City implemented stay-at-home orders and suspended elective surgeries. These stay-at-home orders remained largely in place until June when phased relaxation of stay-at-home restrictions was implemented [3]. Google trend results for "COVID" demonstrate that peak interest since the beginning of 2020 to July was highest on March 29. 


\section{GoogleTrendsresults}

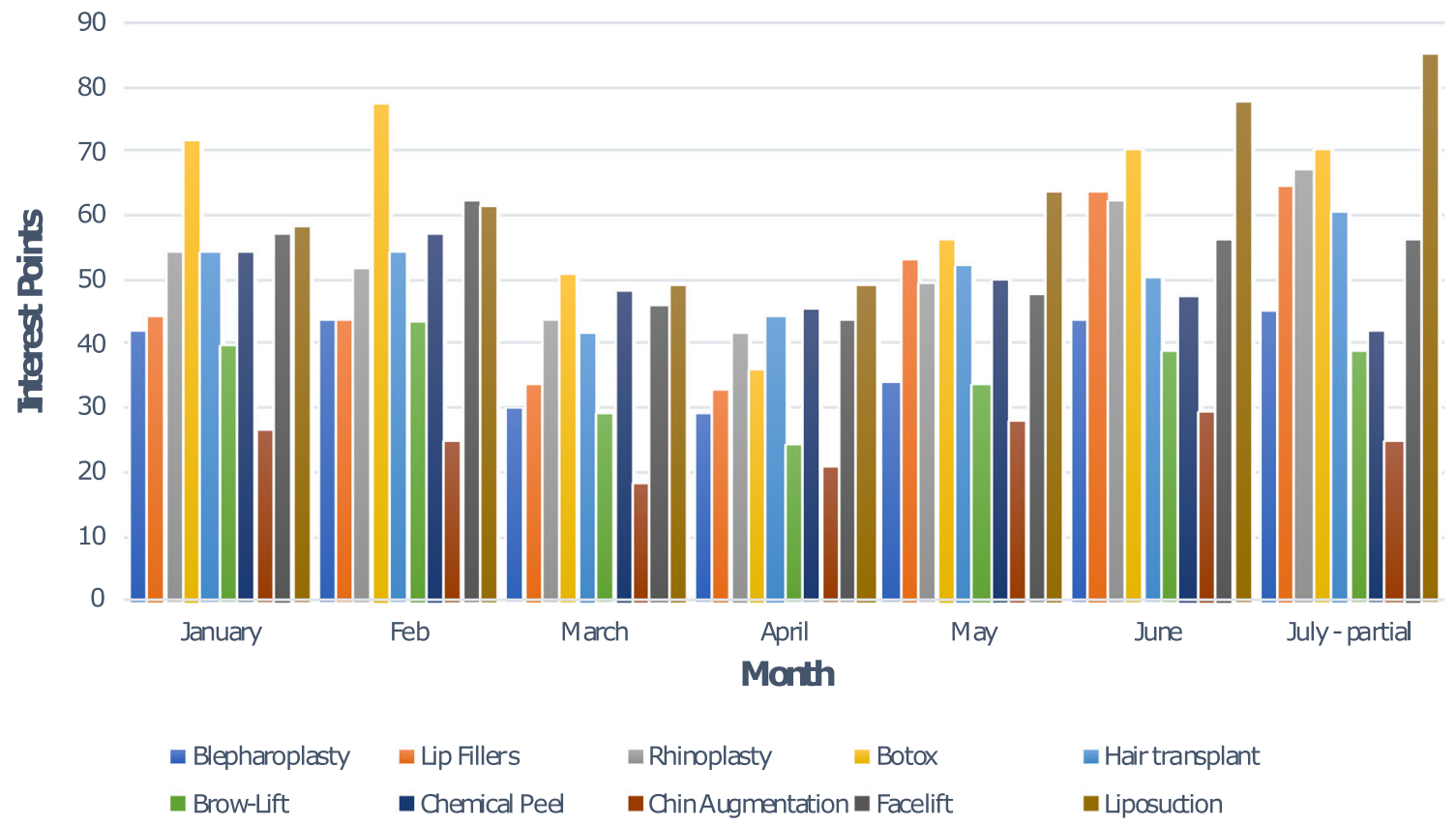

Fig. 1 Google trends interest points for 10 terms broken down by month

Fig. 2 Average interest of all 10 terms displayed by month

Averagesof all Search Terms

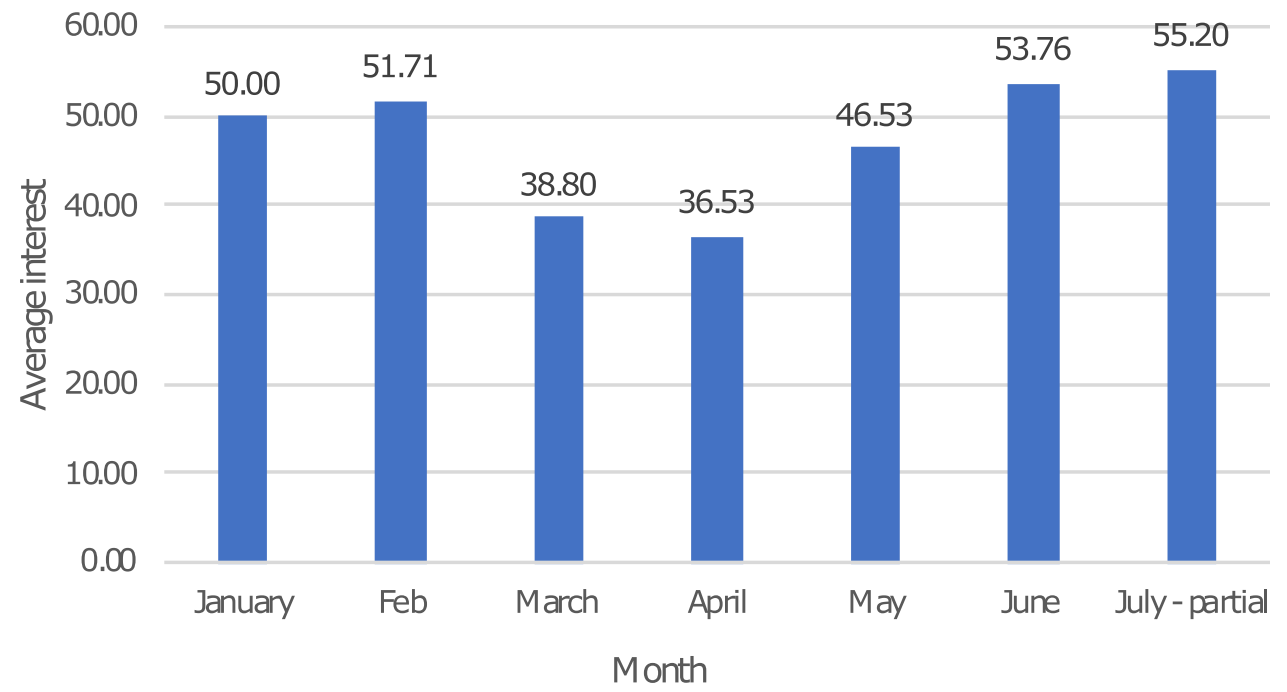

As local lockdowns are lifted, interest in aesthetic and cosmetic procedures is piqued again, even in the context of an ongoing national pandemic. It is possible that as certain sectors of the public move away from their fear of the pandemic and into their "new normal," they once again become interested in nonessential services such as cosmetic surgery.

Many cosmetic procedures have rightfully been put on hold during this pandemic either by patients themselves or their physicians, but physicians should be aware that public interest in aesthetic and cosmetic procedures is as strong as before strict national measures were implemented. This provides an opportunity for physicians to connect with their patients, perhaps providing tele-health consultations or other services until certain that in-person procedures may be performed safely.

Funding There was no source of funding for this article. 


\section{Compliance with Ethical Standards}

Conflict of interest The authors declare that they have no conflicts of interest to disclose.

Ethical Approval Statement of human and animal rights, or ethical approval: This article does not contain any studies with human participants or animals performed by any of the authors.

Informed Consent For this type of study, informed consent is not required.

\section{References}

1. Ward B, Ward M, Paskhover B (2018) Google trends as a resource for informing plastic surgery marketing decisions. Aesthet Plast Surg 42(2):598-602. https://doi.org/10.1007/s00266-017-1019-4

2. Timeline of WHO's response to COVID-19. Accessed July 19, 2020. https://www.who.int/news-room/detail/29-06-2020covidtimeline

3. Timeline: The first 100 days of New York Gov. Andrew Cuomo's COVID-19 response - ABC News. Accessed July 19, 2020. https:// abcnews.go.com/US/News/timeline-100-days-york-gov-andrewcuomos-covid/story?id=71292880

Publisher's Note Springer Nature remains neutral with regard to jurisdictional claims in published maps and institutional affiliations. 